\title{
Swedish Weight Sounding: A prospective portable soil investigation tools for liquefaction assessment of residential houses in Indonesia
}

\author{
Yusa Muhamad $^{1, *}$, Bowman Elisabeth T. ${ }^{2}$, Nugroho, S.A ${ }^{1}$ \\ ${ }^{1}$ Civil Engineering Department, University of Riau, Pekanbaru, 28294, Indonesia \\ ${ }^{2}$ Civil and Structural Engineering, University of Sheffield, Sheffield S13JD, United Kingdom
}

\begin{abstract}
National Disaster Management Agency (BNPB) statistics show that the majority of earthquake affected buildings are residential houses, whereas in practice, soil investigation is rarely conducted for residential houses in Indonesia. This study is preliminary work on the prospective of Swedish Weight Sounding (SWST) for liquefaction assessment for residential houses. Material used is poorly graded sand. The number of half turns from SWST $(\mathrm{Nsw})$ per meter for very loose and loose clean fine sand ranges from 4 to 168 (equivalent to SPT 0-30). Liquefaction potential was assessed using an indirect method by converting $\mathrm{N}_{\text {Sw }}$ into equivalent $\mathrm{N}_{\text {SPT }}$ and direct method. In general, the factor of safety obtained from the direct method is more conservative (thus giving lower liquefaction potential index) than the indirect method. Torque measured for material in this study ranged from 6-54 Nm, equivalent to a specific energy range from 7-70 $\mathrm{N} / \mathrm{mm}^{2}$. Liquefaction assessment using SWST data with torque measurement also indicated the soil is liquefiable. SWST also may be able to detect sand ageing. In summary SWS has a good prospect as a highly portable and low cost investigation tool for liquefaction assessment of residential houses in Indonesia.
\end{abstract}

\section{Introduction}

BNPB [1] shows that, from 1900-2017, earthquake is the deadliest natural disaster in Indonesia. Furthermore, BNPB statistics reveal that earthquake is the most damaging natural disaster, accounting for more than $70 \%$ of damaged houses (Fig. 1).

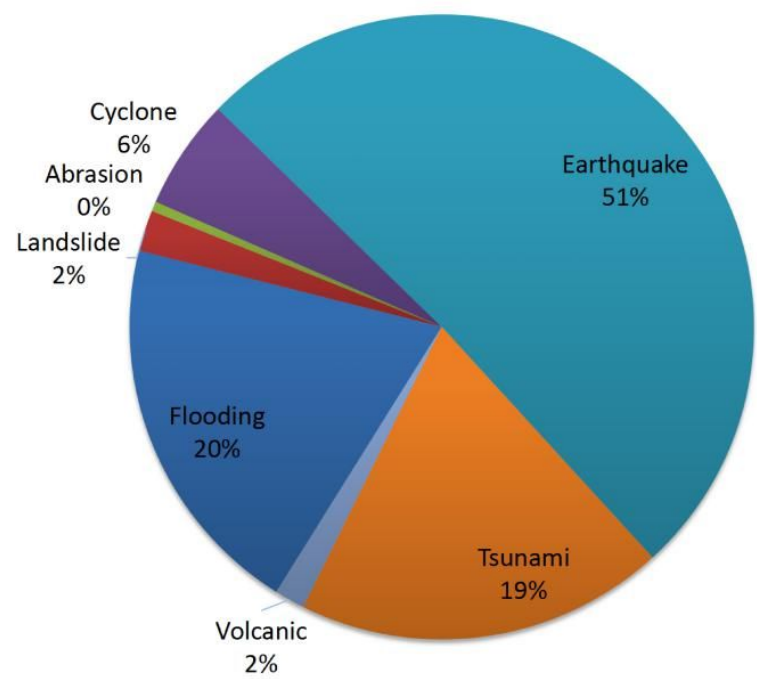

Fig. 1. Damaged houses due to natural disaster (BNPB, 2017)

\footnotetext{
* Corresponding author: m.yusa@eng.unri.ac.id
}

The Palu earthquake in 28 September 2018 with magnitude of 7.4 and $10 \mathrm{~km}$ depth revealed another damaging aspect of earthquakes to be a ground damage i.e. liquefaction. During an earthquake, the sandy soil beneath loses its strength and behaves like a fluid. Although liquefaction has been observed during past earthquakes in Indonesia, the damaging level of the liquefaction in Palu earthquake was unprecedented. Liquefaction not only caused damage to infrastructure and thousands of houses, it also caused thousands of fatalities which did not happen in the previous liquefaction events.

Table 1 shows recorded liquefaction case histories from 1992 to 2018. The table reveals that liquefaction usually occurs when the epicentre is shallow i.e. less than $75 \mathrm{~km}$. It can be seen also that liquefaction not only occurs following large magnitude earthquakes but have happened in the event of earthquakes with magnitude as low as 5 .

\section{Research motivation}

One of the important factors for liquefaction assessment is the local ground condition which requires soil investigation. The common soil investigation tests used in Indonesia are Standard Penetration Test (SPT) and mechanical Cone Penetration Test (CPT). SPT has some advantages i.e. a long history of application and soil type 
can be identified. However, SPT requires heavy machinery, large operational space, and skilled operator and is expensive. SPT also has problems with repeatability. Mechanical CPT requires less operational space, is relatively faster compared to SPT, and gives continuous soil profile but is still operator dependent. In addition, CPT is repeatable and the interpretation has empirical and theoretical basis. However overall both of these common tests are not easily accessible (for residential houses) to many people mainly due to the cost and they are not portable. Experience from earthquake prone countries like Japan and New Zealand shows that liquefaction hazard assessment is required even for low rise/residential building. Following a great number of heavily damage houses associated with liquefaction as in Palu earthquake and previous events, there is need for a cheap (hence easy to be accessed by many people), fast, and portable soil investigation test.

Table 1. Indonesia Liquefaction case histories 1992-2018

\begin{tabular}{|c|c|c|c|}
\hline No & Location & M & Depth \\
\hline 1 & Maumere(1992) & 6.8 & 35 (sea) \\
\hline 2 & Bengkulu(2000) & 7.3 & 33 (sea) \\
\hline 3 & Aceh(2004) & 9.2 & 30 (sea) \\
\hline 4 & Nias(2005) & 8.7 & 30 (sea) \\
\hline 5 & Jogjakarta(2006) & 5.9 & 11.3 (land) \\
\hline 6 & Cilacap(2006) & 7.7 & $48.6($ sea) \\
\hline 7 & Solok(2007) & 6.4 & $30($ land) \\
\hline 8 & Bengkulu(2007) & 8.4 & 10 (sea) \\
\hline 9 & Tasikmalaya(2009) & 7.3 & 49 (sea) \\
\hline 10 & Padang(2009) & 7.6 & 71 (sea) \\
\hline 11 & Mentawai(2010) & 7.7 & 14.2 (sea) \\
\hline 12 & Poso(2017) & 6.6 & 10 (land) \\
\hline 13 & Poso(2017) & 5.1 & $10($ sea) \\
\hline 14 & Lombok(2018) & 6.4 & $24($ land) \\
\hline 15 & Lombok(2018) & 6.9 & 24(land) \\
\hline 16 & Palu(2018) & 7.5 & 10 (land) \\
\hline
\end{tabular}

There are some highly portable and low cost (HP-LC) soil investigation tools available in the market such as handheld cone penetrometer (HCP), macintosh probe (MCP) and dynamic cone penetrometer (DCP). However, the maximum penetration depth is typically 3 meters whereas the liquefaction depth can be up to $10-15 \mathrm{~m}$ below ground surface. Learning from Japan, New Zealand and Iran, the Swedish Sounding Test (SWST) has been used extensively for field reconnaissance following an earthquake and as a soil investigation tool (to about $10 \mathrm{~m}$ depth) for low rise buildings [e.g. 2-4]. SWST mainly consists of a screw point, rods, a rotating handle and the weight (Fig. 2). According to British standard EN ISO 22476-10:2017, what is measured is the number half turns for $20 \mathrm{~cm}$ penetration under $100 \mathrm{~kg}$ weight (detailed procedure is given in 2.1.3). Using the Japanese Industrial
Standard (JIS) 1221-2013, the measurement is taken at intervals of $25 \mathrm{~cm}$.

This research is a preliminary work regarding the use of SWST as prospective HP-LC soil investigation tools for liquefaction assessment of residential houses in Indonesia.

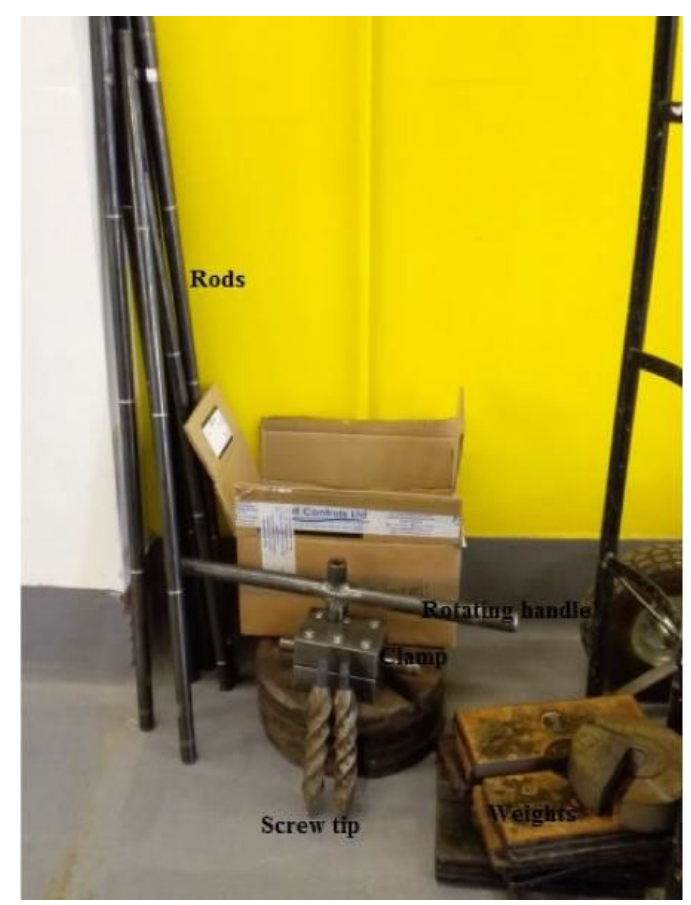

Fig. 2. SWST apparatus

\section{Material, equipment and location}

Soils used in this research were selected to have similar properties as the soil from western coastal area of Sumatra such as Padang i.e. clean sand and silty sand. Hakam and Darjanto [6] reported that ejected sand material from Padang Earthquake mainly consists of more than $60 \%$ fine sand and fines content less than $20 \% . \mathrm{d}_{50}$ ranges from $0.15-0.35 \mathrm{~mm}$. Thus for this study, similar highly probable liquefaction material was chosen with poor gradation and $\mathrm{d}_{50}$ of 0.21 (Fig. 3). The material properties used in this study is shown in Table 2.

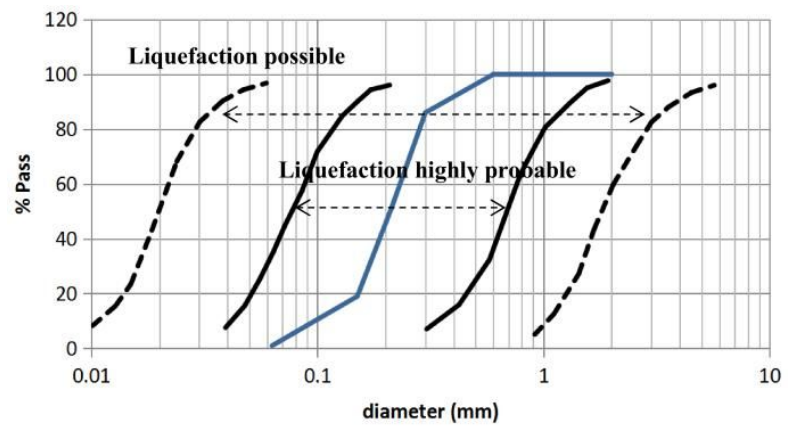

Fig. 3. Material used in this study 
Table 2. Material properties

\begin{tabular}{|l|l|c|}
\hline No & \multicolumn{1}{|c|}{ Parameter } & Value \\
\hline 1 & Water content, w(\%) & 7.6 \\
\hline 2 & Specific gravity & 2.68 \\
\hline 3 & Gravel Fraction & 0 \\
\hline 4 & Sand Fraction (\%) & 99 \\
\hline 5 & Fines Fraction (\%) & 1 \\
\hline 6 & $\mathrm{D}_{60}$ & 0.23 \\
\hline 7 & $\mathrm{D}_{50}$ & 0.21 \\
\hline 8 & $\mathrm{D}_{30}$ & 0.17 \\
\hline 9 & $\mathrm{D}_{10}$ & 0.1 \\
\hline 10 & $\gamma_{\mathrm{dmax}}\left(\mathrm{t} / \mathrm{m}^{3}\right)$ & 1.64 \\
\hline 11 & $\gamma_{\text {dmin }}\left(\mathrm{t} / \mathrm{m}^{3}\right)$ & 1.36 \\
\hline 12 & $\mathrm{w}_{\text {optimum }}(\%)$ & 16 \\
\hline 13 & $\phi($ degree $)$ & 33 \\
\hline 14 & Cohesion $(\mathrm{kPa})$ & 1 \\
\hline 15 & Permeability $(\mathrm{m} / \mathrm{s})$ & $1.5 .10^{-6}$ \\
\hline 16 & $\mathrm{pH}$ & 8.4 \\
\hline
\end{tabular}

The tests were conducted at new The Integrated Civil and Infrastructure Research Centre (ICAIR) facility at University of Sheffield, UK. The sand box (Fig. 4) is a box of about $40 \mathrm{~m}$ length, $6 \mathrm{~m}$ width and $5 \mathrm{~m}$ depth as can be seen in Fig. 4. Sand filling process (up to $4.5 \mathrm{~m}$ thickness) was conducted using small excavator. The total volume sand required was about 2000 ton. Due to the large volume of the sand and installation of pipes and instrumentation, the filling process took about 1 month. Seven SWS tests were conducted at several locations in the box (Fig. 5) adjacent to the pipes.

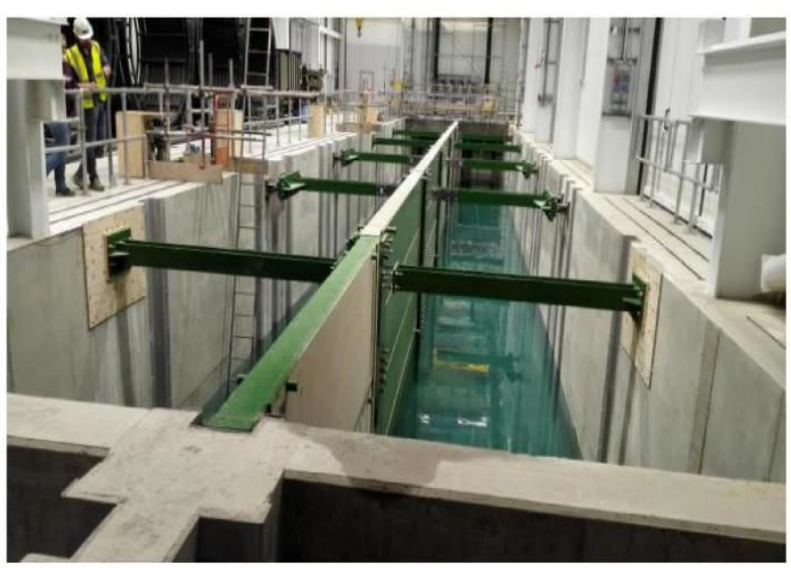

Fig. 4. Sand box

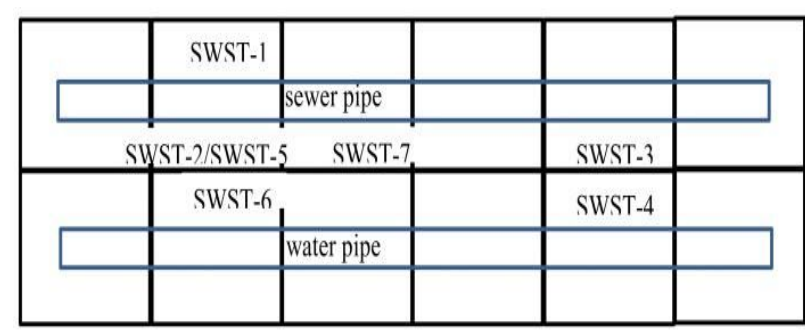

Fig. 5. Test location

\section{Results and Analysis}

\subsection{Results}

Fig. 6(a) shows results from the SWST in terms of number of half turns, $\mathrm{N}_{\mathrm{SW}}$. Number of half turns is converted into per $\mathrm{m}$ instead of per $25 \mathrm{~cm}$ using the equation $\mathrm{N}_{\mathrm{SW}}$ (per $\mathrm{m})=100 /$ (penetration length in $\mathrm{cm}) * \mathrm{~N}_{\mathrm{SW}}$ (per $25 \mathrm{~cm}$ ). Based on Inada equation [7],

$$
N_{S P T}=2 W_{s w}+0.067 N_{s w}
$$

in general $\mathrm{N}_{\mathrm{SW}}$ (per $\mathrm{m}$ ) for the uncompacted sand (very loose to loose) ranges from 4 to 168 . The only exception is $\mathrm{N}_{\mathrm{SW}}$ from SWST-3 at $4 \mathrm{~m}$ depth i.e. $\mathrm{N}_{\mathrm{SW}}$ (per $\left.\mathrm{m}\right)=400$ which indicated medium dense sand.

Fig. 6(b) shows results of SWST with additional torque measurement. An increment of work done by torque and weight is defined as [8]

$$
\delta E=\pi T \delta n_{h t}+W \delta s_{t}
$$

where $\mathrm{E}=$ work done; $\mathrm{T}=$ measured torque; $\delta \mathrm{n}_{\mathrm{ht}}=$ incremental number of half turns; $\mathrm{W}=$ weight; $\delta \mathrm{s}_{\mathrm{t}}=$ incremental settlement caused by load. Specific Energy is defined as the amount of energy divided by volume of penetration.

$$
E S=\frac{E}{L A}
$$

where $\mathrm{L}$ is the amount of penetration and $\mathrm{A}$ is the maximum cross section of the screw tip (i.e. diameter $=$ $35 \mathrm{~mm}^{2}$ ).

\subsection{Analysis}

\subsubsection{Equivalent SPT}

Based on Inada's equation, the equivalent NSPT values were obtained and are shown in Fig. 7. The Fig. generally indicates that the sand condition up to $2.75 \mathrm{~m}$ depth is very loose $\left(\mathrm{N}_{\mathrm{SPT}}<4\right)$, followed by loose $\left(4<\mathrm{N}_{\mathrm{SPT}}<10\right)$ up to $3.25 \mathrm{~m}$ depth then very loose again up to $4 \mathrm{~m}$ depth. The exception is the result from SWST-3 and SWST-4 which indicates medium dense condition from 3.25/3.5-4 m depth.

\subsubsection{Estimation of soil type}

Tanaka (2007) proposed estimation of soil type from SWT with torque measurement based on Equation 4:

$$
N_{s w} D=c_{p} \frac{\pi T}{W D}
$$

where $\mathrm{D}$ is the diameter of the screw point, $\mathrm{T}$ is torque and $\mathrm{W}$ is weight. Tanaka [9] reported that values of $c_{p}$ can be 
used to classify the soil type. Table 3 shows guidance for the estimation of soil type using SWST with torque measurement.

Table 3. Soil type estimation from SWST [8]

\begin{tabular}{|l|l|}
\hline$c_{p}$ & Soil type \\
\hline$>0.1$ & sand \\
\hline $0.3<c_{p}<0.1$ & Clay/silt \\
\hline$<0.3$ & organic \\
\hline
\end{tabular}

$\mathrm{N}_{\mathrm{sw}}($ half tum $/ \mathrm{m})$

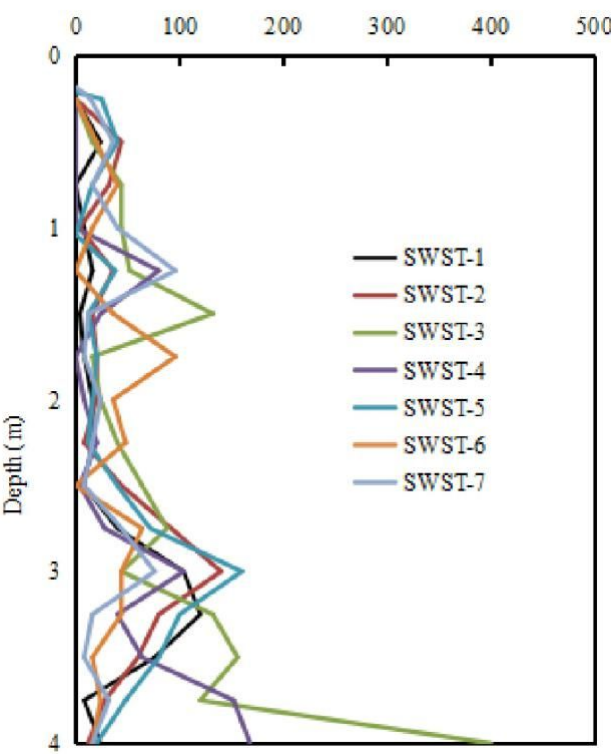

(a)

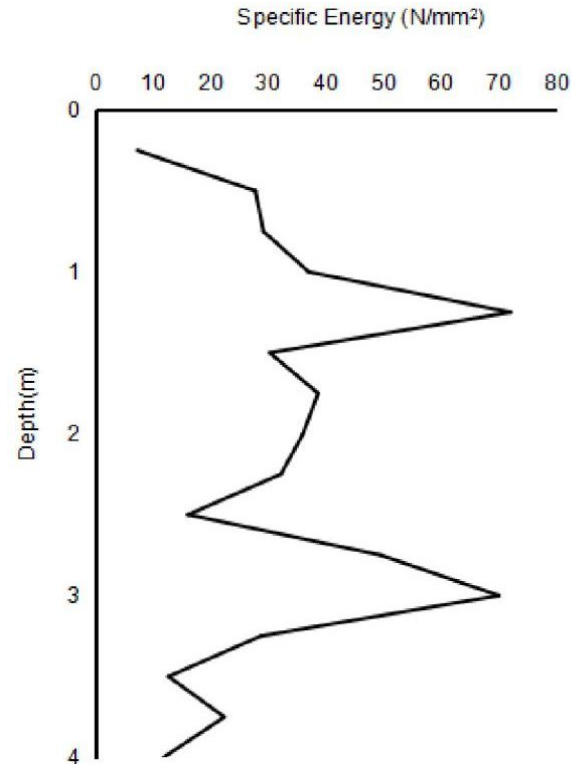

(b)

Fig. 6. SWST results (a) $\mathrm{N}_{S w}$ (b)Specific Energy, Es

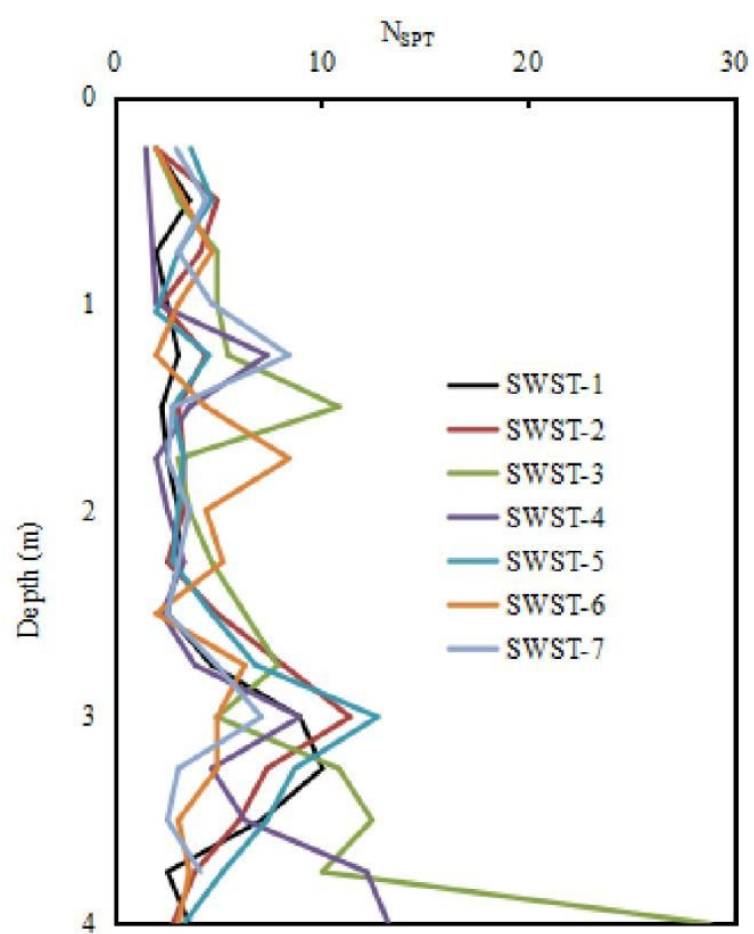

Fig. 7. Equivalent SPT

Based on Table 3 and SWST-7 results it can be seen from Table 4 that all $c_{p}$ values up to $4 \mathrm{~m}$ depth $>0.1$ indicate sand, which is the case.

Table 4. Soil type estimation from SWST-7

\begin{tabular}{|c|c|c|c|}
\hline Depth & Torque $(\mathrm{Nm})$ & $c_{p}$ & Soil Est. \\
\hline 0.25 & 11.0 & 28.2 & Sand \\
\hline 0.5 & 28.4 & 8.1 & Sand \\
\hline 0.75 & 24.5 & 15.7 & Sand \\
\hline 1 & 26.5 & 6.8 & Sand \\
\hline 1.25 & 48.5 & 5.2 & Sand \\
\hline 1.5 & 17.5 & 15.0 & Sand \\
\hline 1.75 & 24.0 & 30.8 & Sand \\
\hline 2 & 21.6 & 9.2 & Sand \\
\hline 2.25 & 17.1 & 10.9 & Sand \\
\hline 2.5 & 8.5 & 10.9 & Sand \\
\hline 2.75 & 29.0 & 6.8 & Sand \\
\hline 3 & 44.0 & 5.9 & Sand \\
\hline 3.25 & 26.5 & 17.0 & Sand \\
\hline 3.5 & 14.0 & 18.0 & Sand \\
\hline 3.75 & 34.1 & 10.9 & Sand \\
\hline 4 & 20.8 & 13.3 & Sand \\
\hline
\end{tabular}




\subsubsection{Liquefaction assessment}

Basically there are two factors needed i.e. cyclic stress ratio (CSR) which represents the load due to earthquake and cyclic resistance ratio (CRR) which represents the dynamic soil strength (obtained from soil investigation e.g. SPT, CPT, SWST). The Factor of safety against liquefaction is defined as the ratio between CRR and CSR.

CRR was calculated by two methods i.e. indirectly based on SPT using Iwasaki Method [10] and directly based on SWST [8].

$$
\begin{aligned}
& \operatorname{CSR}=0.65(0.1)(\mathrm{M}-1)(1-0.015 \mathrm{z}) \sigma_{\mathrm{v}} / \sigma_{\mathrm{v}}^{\prime} \\
& C R R=0.0882 \sqrt{\frac{N_{S P T}}{0.7+\sigma_{v}^{\prime}}}+0.225 \log \frac{0.35}{D_{50}}
\end{aligned}
$$

For $\mathrm{a} \leq 0.3 \mathrm{~g}$ and clean sand

$$
C R R=0.016 \sqrt{N_{S W 1}^{\prime}} ;
$$

For $\mathrm{a} \leq 0.3 \mathrm{~g}$ and silty sand

$$
\begin{gathered}
C R R=0.02 \sqrt{N_{S W 1}^{\prime}} \\
\mathrm{N}^{\prime}{ }_{S W}=\mathrm{N}_{S W}+40 \\
N_{S W 1}^{\prime}=N_{S W}^{\prime} \sqrt{\frac{98}{\sigma_{v}^{\prime}}}
\end{gathered}
$$

It was assumed that the peak surface acceleration is $0.3 \mathrm{~g}$ and earthquake magnitude of 7.6, similar to the Padang earthquake of 2009. Ground water level is assumed $1 \mathrm{~m}$ below the ground surface as the ground water level at Padang City ranges from $0-3 \mathrm{~m}$. Analysis of results is exemplified in Fig. 8. The Fig.s show that the majority of FS values with depth, calculated using both methods, are less than 1 (potentially liquefiable).

Further analysis was conducted using qualitative Liquefaction Potential Index (LPI) classification [11].

$$
L P I=\int_{0}^{z c r} F(z) w(z) d z
$$

where $w(z)=\frac{200}{z c r}\left(1-\frac{z}{z c r}\right)$ is a weighting factor that gives greater influence to shallower layer and $F(z)$ is factor of safety for each layer. For soil profile less than $20 \mathrm{~m}$, LPI can be calculated using the following equations [12]:

$$
\mathrm{LPI}=\sum_{i=1}^{n} \omega_{i} F_{i} H_{i}
$$

where $\mathrm{Hi}$ is the layer thickness; $\mathrm{Fi}=1-\mathrm{FSi} ; \mathrm{FS}<1.0$; Fi $=0 ; \mathrm{FS}>1.0$. The SWST tests give LPI values higher than 5 and 15 indicating high to very high or moderate to major potential of liquefaction as presented in Table 5. It should be noted that the direct method is based on limited case histories in Japan. Development of a further database based on real cases in Indonesia is required.

Liquefaction assessment using SWST with torque measurement based on Mirjafari's work [13] is shown in Fig. 9. The Fig. shows that the majority of the depth potentially liquefies except at depth $1.25 \mathrm{~m}$ and $3 \mathrm{~m}$ which lie to the right of the curve.

\subsubsection{Ageing study based on SWST}

Test SWST-5 was conducted about two weeks after SWST-2, to investigate whether ageing can be detected using SWST. To minimize the effect of soil variation SWST-5 was conducted only $30 \mathrm{~cm}$ from SWST-2 location. Fig. 9 shows comparison between the two tests. The Fig. shows that for soils deeper than 3 meter SWST5 results is slightly higher $6-13 \%$ ( $11 \%$ on average) than SWST-2 results, indicating possibility of aging effect. However, it is possible also that the increase may be due to other factors. More tests are needed to confirm whether SWST can detect ageing.

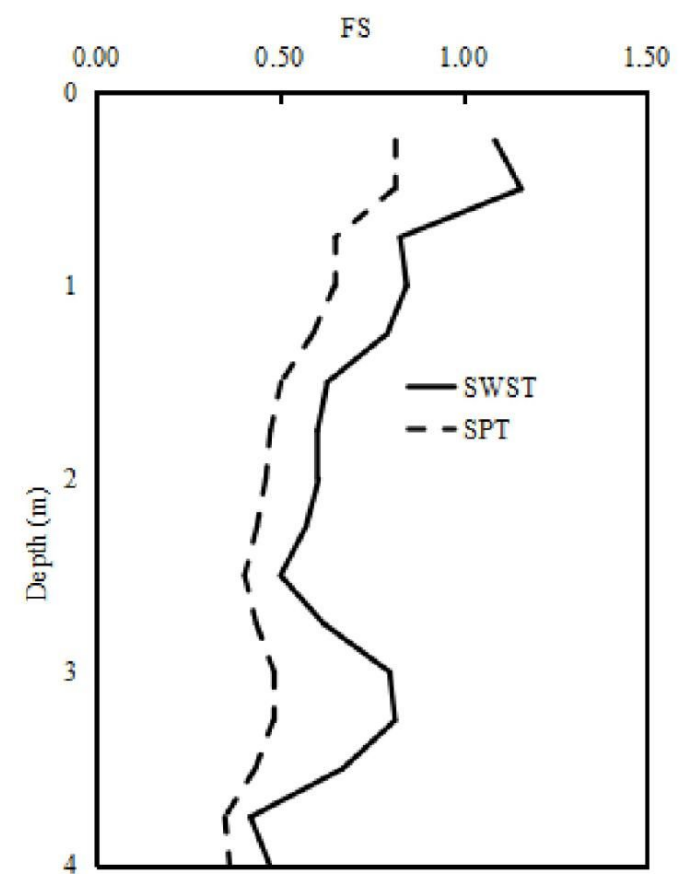

Fig. 8. Depth vs FS (SWST-1)

Table 5. Liquefaction Potential Index

\begin{tabular}{|c|c|c|c|}
\hline \multirow{2}{*}{ No } & \multicolumn{2}{|c|}{ LPI } & \multirow{2}{*}{ Note } \\
\cline { 2 - 3 } & SWST & SPT $* n$ & \\
\hline SWST-1 & 9.7 & 15.9 & $0=$ very low \\
\hline SWST-2 & 8.4 & 15.4 & $0<$ LPI $<5$ :low \\
\hline SWST-3 & 5.21 & 12.9 & $5<$ LPI $<15$ :high \\
\hline SWST-4 & 10.4 & 18.8 & $>15$ :very high \\
\hline SWST-5 & 7.6 & 15.6 & \\
\hline SWST-6 & 9.4 & 22 & \\
\hline SWST-7 & 10.2 & 16.4 & \\
\hline
\end{tabular}




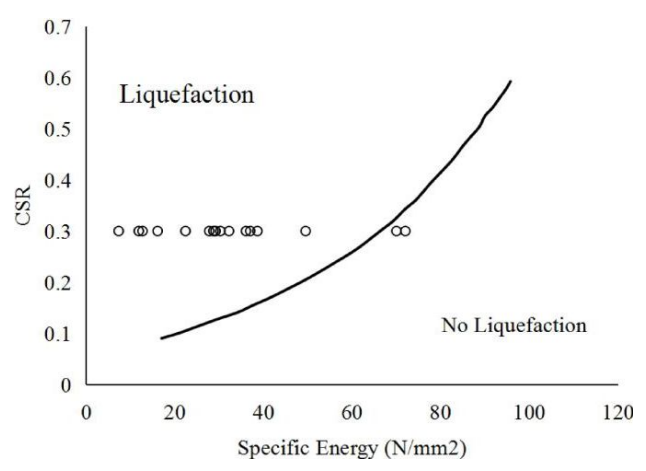

Fig. 9. Liquefaction assessment using SWST with torquemeter

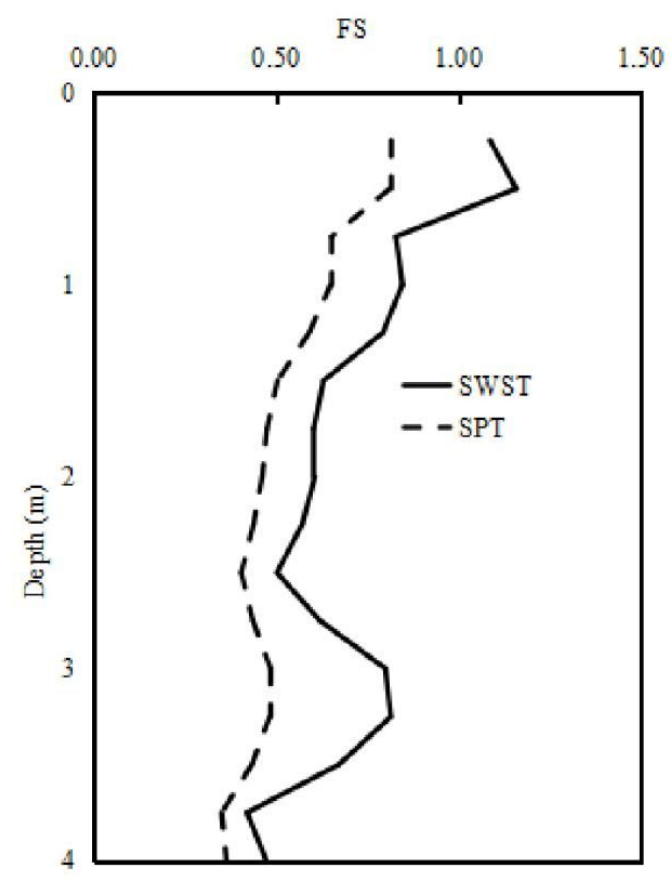

Fig. 10. Ageing study

\section{Conclusions}

In general, SWST with and without torque measurement has high potential to be used for liquefaction assessment for residential houses in small-medium cities in Indonesia mainly due its portability, ease of operation and ability to reach liquefaction depth. In addition, as the results of further literature studies and analysis some conclusions can be drawn as follows:

1. Number of half turns per meter for very loose and loose clean fine sand ranges from 4 to 168 .

2. Liquefaction assessment using SPT equivalent and direct SWST method shows that the majority of the soil depth up to $4 \mathrm{~m}$ has factor of safety against liquefaction less than 1.

3. Generally, liquefaction assessment using direct SWST data as proposed by Tsukamoto et al (2016) gave a lower factor of safety than the SPT method (Iwasaki, 1982). However, it should be noted that the direct SWST method is still based on very limited case histories in Japan.

4. Liquefaction assessment reveals high to very high or moderate to major liquefaction potential index (LPI) with direct manual SWST method giving lowest LPI values.

5. SWST with torque measurement may be used to identify soil type better compared to hearing the sound during penetration. Torque range for very loose to loose sand in this study ranged from 6-54 Nm, equivalent to specific energy range from $7-70 \mathrm{~N} / \mathrm{mm}^{2}$. Liquefaction assessment using SWST data with torque measurement also indicated the soil is liquefiable.

6. Based on SWST-2 and SWST-5, SWST might be able to detect ageing but more tests are required to conform it.

The Authors greatly thank Newton Fund Research Link Travel Grant ID 2017-RLTG9-10615 which funded the work at University of Sheffield, UK.

\section{References}

1. BNPB. Data Information Indonesia Disaster. Retrieved Date Accessed, 2017 from http://bnpb.cloud/dibi//tabel1b. (2017).

2. Onoue, A., \& Toyota, H. Proc. 14th World Conference on Earthquake Engineering, (2008)

3. Taylor, M., \& Cubrinovski, M. Earthq Engg Buildg and Earthquake-Resilient Society (2011)

4. Habibi, M., Cheshomi, A., \& Fakher, A. Proc. 4th International Conference on Earthquake Engineering, (2006)

5. JIS. JIS A 1221 Japanese Industrial Standard. Method of Swedish Weight Sounding Rev. 4: Japanese Standards Association (1995).

6. Hakam, A., \& Darjanto, H. Teoretical and Applied Civil Engineering, 20(1), pp. 33-38, (2013).

7. Inada, M. Use of Swedish weight sounding tests. Tsuchi-to-Kiso, 8(1), 13-18, (1960).

8. Tsukamoto, Y., Hyodo, T., \& Hashimoto, K. J. Soils and Foundations, 56(1), pp. 104-114, (2016).

9. Tanaka, T., Suemasa, N., Yamato, S., \& Katada, T. Proceedings of the Seventeenth International Offshore and Polar Engineering Conference (2007)

10. Iwasaki, T., Tokida, K., \& Tatsuoka, F. Proc. International Conference on Recent advance geotechnical earthquake engineering and soil dynamic (1981)

11. Iwasaki, T., Tokida, K., \& Tatsuoka, F. Proc. International Conference on Recent advance geotechnical earthquake engineering and soil dynamic (1981)

12. Luna, R., \& Frost, J. D. Computing Civil Engineering, 12 48-56 (1998)

13. Mirjafari, S. Y., Orense, R., \& Suemasa, N. New Zealand Soc. of Earthq. Engg (2014) 